\title{
Impaired cardiac autonomic nervous system function is associated with pediatric hypertension independent of adiposity
}

\author{
Justin R. Ryder ${ }^{1,2}$, Michael O'Connell ${ }^{3}$, Tyler A. Bosch ${ }^{4}$, Lisa Chow ${ }^{4}$, Kyle D. Rudser ${ }^{3}$, Donald R. Dengel ${ }^{1,5}$, Claudia K. Fox ${ }^{1}$, \\ Julia Steinberger ${ }^{1}$ and Aaron S. Kelly,4
}

BACKGROUND: We examined whether sympathetic nervous system activity influences hypertension status and systolic blood pressure (SBP) independent of adiposity in youth ranging from normal-weight to severe obesity.

METHODS: We examined the association of heart rate variability (HRV) with hypertension status and SBP among youth (6-18 y old; $n=188 ; 103$ female). Seated SBP was measured using an automated cuff. Prehypertension (SBP percentile $\geq$ 90th to <95th) and hypertension (SBP percentile $\geq$ 95th) were defined by age-, sex-, and height-norms. Autonomic nervous system activity was measured using HRV via SphygmoCor MM3 system and analyzed for time- and frequency-domains. Total body fat was measured via dual-energy X-ray absorptiometry. RESULTS: Logistic regression models demonstrated lower values in each time-domain HRV measure and larger lowfrequency (LF):high-frequency (HF) ratio to be significantly associated with higher odds of being prehypertensive/hypertensive (11-47\% higher odds) independent of total body fat $(P<0.05)$. In linear regression analysis, lower time-domain, but not frequency-domain, HRV measures were significantly associated with higher SBP independent of total body fat $(P<0.05)$.

CONCLUSION: These data suggest that impaired cardiac autonomic nervous system function, at rest, is associated with higher odds of being prehypertensive/hypertensive and higher SBP which may be independent of adiposity in youth.

$\mathrm{n}$ adults, prehypertension and hypertension are associated with increased risk of cardiovascular disease (CVD) mortality (1). In childhood, prehypertension and hypertension track into adulthood (2), potentially compounding the lifetime CVD burden (3). Many of the pathophysiological processes contributing to essential hypertension in adults have become clear over the past few decades (4). However, due to the relatively low proportion of children with hypertension (5), much work still remains to elucidate whether the same contributors are operational in childhood.

Cardiac autonomic nervous system (cANS) function and sympathetic tone have been shown to have a strong influence on the regulation of arterial blood pressure (BP) (6). The activity of the cANS can be measured noninvasively using heart rate variability (HRV), which measures beat-to-beat variations in the cardiac cycle (7). HRV can be subdivided into time- and frequency-domains with multiple measures within each domain. Each time- and frequency-domain may have a different physiological meaning and therefore may represent unique variables of interest (8). Under resting conditions, time-domain measures of HRV represent differences in beatto-beat control mechanisms largely regulated by sympathetic and vagal efferent activity as well as central oscillators (i.e., respiratory movements) (9). Frequency-domains can be split into high-frequency (HF) and low-frequency (LF) partitions. $\mathrm{HF}$ is likely indicative of parasympathetic nervous system modulation of cardiac function, while LF is indicative of primarily sympathetic nervous system modulation with some influence from the parasympathetic nervous system $(8,10)$. Together, time- and frequency-domain measures provide a complete picture of cANS fluctuations and control. In adults, time- and frequency-domain measures of HRV are predictive of CVD and future CVD events (i.e., myocardial infarction and stroke) $(11,12)$.

In youth, impaired HRV is associated with physical inactivity (13), low cardiovascular fitness (14), and endothelial dysfunction (15). Recently, Farah et al. (16) provided evidence that both time- and frequency-domain measures of HRV were related to multiple CVD risk factors in youth, including systolic blood pressure (SBP). However, the role of adiposity in mediating these relationships was not investigated despite its potential physiological relevance. Although obesity has increased over the past 30 years, secular trends in

\footnotetext{
'Department of Pediatrics, University of Minnesota Medical School, Minneapolis, Minnesota; ${ }^{2}$ Division of Epidemiology \& Community Health, University of Minnesota School of Public Health, Minneapolis, Minnesota; ${ }^{3}$ Division of Biostatistics, University of Minnesota School of Public Health, Minneapolis, Minnesota; ${ }^{4}$ Department of Medicine, University of Minnesota Medical School, Minneapolis, Minnesota; ${ }^{5}$ School of Kinesiology, University of Minnesota, Minneapolis, Minnesota. Correspondence: Justin R. Ryder (jrryder@umn.edu)

Received 21 April 2015; accepted 21 July 2015; advance online publication 14 October 2015. doi:10.1038/pr.2015.188
} 


\section{Articles Ryderetal.}

SBP among children remained stable (5), suggesting obesity may not be directly related to higher SBP in youth. While several cross-sectional studies have shown obesity and excess adiposity to be associated with higher SBP, prehypertension, and hypertension in children and adolescents (17-19), these studies did not account for the association between adiposity and HRV, which has been previously described in youth $(15,20-22)$. Since adiposity likely influences both HRV and SBP regulation, characterizing the relationships among these variables has important physiological relevance.

Therefore, the purpose of this study was to examine the relationship of cANS function (as measured by HRV time- and frequency-domains) with hypertension status and level of SBP independent of adiposity in youth ranging from normalweight to severe obesity.

\section{RESULTS}

A higher proportion of females were prehypertensive and hypertensive (Table 1). Hypertensive participants were all overweight/obese or severely obese. Caucasian race was predominant across all groups with a trend toward the hypertensive group having a higher minority presence. The groups had similar distributions of pubertal maturation levels as determined by Tanner stage.

Lower values of every time-domain HRV measure except NN50 were associated with significantly higher odds of being prehypertensive/hypertensive adjusted for Tanner stage, race, and total body fat $(P<0.05$ all; Table 2$)$. Lower levels of mean R-R interval length for each $50 \mathrm{~ms}$ increment were associated with a $33 \%$ higher odds of being prehypertensive/hypertensive; 10 ms lower SD between R-R intervals (SDRR) was associated with a $11 \%$ higher odds of being prehypertensive/hypertensive; $10 \mathrm{~ms}$ lower corrected SDRR was associated with a $41 \%$ higher odds of being prehypertensive/ hypertensive; $10 \mathrm{~ms}$ lower root mean square of the square difference between adjacent normal R-R intervals (RMSSD) was associated with a $11 \%$ higher odds of being prehypertensive/hypertensive; and each 10 unit difference in pNN50 was associated with a $21 \%$ higher odds of being prehypertensive/hypertensive. Higher LF:HF ratio during rest resulted in a $47 \%$ higher odds of being prehypertensive/hypertensive $(P=0.011)$. Consistent with the LF:HF ratio were the trends in associations of LF and HF normalized units $(P=0.07$ for both).

Table 3 shows linear regression analyses examining the association between each time- and frequency-domain HRV measure with SBP adjusting for Tanner stage, race, age, sex, height, and total body fat. Lower values for each HRV timedomain variable (mean, SDRR, corrected SDRR, RMSSD, NN50, pNN50) were significantly associated with higher SBP independent of adiposity $(P<0.05$ all $)$. None of the frequencydomain HRV variables (LF normalized, HF normalized, or LF:HF ratio) were significantly associated with higher SBP. When smokers, current or past $(n=5)$, were removed from all analysis, the results did not differ.

\section{DISCUSSION}

These findings demonstrate a consistent association between impaired autonomic nervous system control, at rest, with higher odds of prehypertension/hypertension and higher SBP among youth. Importantly, these associations were found to be independent of total body fat. Overall, these data suggest an adverse shift in sympathovagal balance in youth with prehypertension/hypertension. Furthermore, while hypertensive youth were more likely to be obese, the impairment in autonomic nervous system function was independent of adiposity.

Our results shed light on the relationship of cANS function, as measured by HRV, with hypertension status in youth. We observed smaller time-domain measures of HRV (Mean R-R, SDRR, RMSSD, corrected SDRR, and pNN50) and elevated frequency-domain measures of HRV (LF:HF) to be associated with higher odds of prehypertension/hypertension. The higher levels of LF:HF ratio and lower time-domain HRV measures, specifically RMSSD, corrected SDRR and mean R-R, are indicative of increased sympathetic modulation or decreased parasympathetic activity leading to impairment of cardiac function. Additionally, we observed lower levels of all time-domain measures of HRV (Mean R-R, SDRR, RMSSD, Corrected SDRR, NN50 and pNN50) but no frequency-domain measures of HRV to be associated with higher SBP. The lack of association between SBP and frequency-domain variables may be due to the fact that the latter were measured under resting conditions, which likely limit the variability and range of values making it difficult to detect associations with SBP.

Recent data from Farah et al. (16), show similar associations between time-domain HRV measures and SBP. However, they observed a significant association between several frequencydomain HRV measures and higher SBP, which are at odds with our data. A potential explanation may be the differences in methods used, as our study utilized SphygmoCor MM3 system while the Farah et al., used a heart rate monitor (POLAR, RS 800CX), which may have differences in sensitivity. Another potential explanation for this discrepancy is our adjustment for pubertal maturation and adiposity, both of which have been shown to affect these relationships $(23,24)$. Additionally, our sample had a larger proportion of youth classified as prehypertensive/hypertensive ( 25 vs. $9.7 \%$ ), which could help explain these differences.

Importantly, our data demonstrate a strong association between measures of HRV with odds of being prehypertensive/hypertensive and with higher SBP, even after accounting for adiposity. In adults, there is a clear association between body fat, regardless of type or region, and higher SBP. Data from the Framingham Heart Study demonstrated significant associations of higher amounts of VAT and subcutaneous adipose tissue (SAT) with higher SBP in both men and women (25). Moreover, data from Framingham suggest that approximately $65-75 \%$ of the risk for hypertension in adults can be attributed to excess adiposity (26). However, in children, secular trends show no increase in SBP among children despite higher obesity prevalence rates over the same period of time 
Table 1. Participant demographic and clinical characteristics split by hypertension status

\begin{tabular}{|c|c|c|c|c|}
\hline & Normotensive & Prehypertensive & Hypertensive & \\
\hline Covariates & $N=141$ & $N=16$ & $N=31$ & $P$ value \\
\hline Female & $73(51.8 \%)$ & $11(68.8 \%)$ & $19(61.3 \%)$ & 0.038 \\
\hline Height $(\mathrm{cm})$ & $157(13.2)$ & $157(12.8)$ & $158(12.7)$ & 0.119 \\
\hline Weight (kg) & $66.9(25.1)$ & $76.3(28.5)$ & $87.7(25.4)$ & $<0.001$ \\
\hline BMI percentile (\%) & $81.0(25.1)$ & $89.0(24.2)$ & $98.7(0.75)$ & $<0.001$ \\
\hline Race & & & & 0.499 \\
\hline Asian & $2(1.4 \%)$ & $1(6.2 \%)$ & $3(9.7 \%)$ & \\
\hline African American & $14(9.9 \%)$ & $1(6.2 \%)$ & $5(16.1 \%)$ & \\
\hline White & 111 (78.7\%) & $13(81.2 \%)$ & $18(58.1 \%)$ & \\
\hline$\|$ & $31(22.0 \%)$ & $4(25.0 \%)$ & $7(22.6 \%)$ & \\
\hline III & $28(19.9 \%)$ & $4(25.0 \%)$ & $5(16.1 \%)$ & \\
\hline IV & $33(23.4 \%)$ & $3(18.8 \%)$ & $7(22.6 \%)$ & \\
\hline V & $17(12.1 \%)$ & $2(12.5 \%)$ & $5(16.1 \%)$ & \\
\hline Heart rate (bpm) & $73.5(11.4)$ & $75.4(7.3)$ & $80.7(10.1)$ & 0.082 \\
\hline $\mathrm{SBP}(\mathrm{mmHg})$ & $111(9.15)$ & $123(5.75)$ & $135(8.35)$ & $<0.001$ \\
\hline $\mathrm{DBP}(\mathrm{mmHg})$ & $57.5(7.51)$ & $62.9(5.83)$ & $65.7(9.54)$ & 0.003 \\
\hline SBP percentile (\%) & $55.3(23.4)$ & $92.2(1.47)$ & $98.5(1.67)$ & $<0.001$ \\
\hline SDRR (ms) & $83.2(41.0)$ & $74.3(23.0)$ & $63.7(29.1)$ & 0.313 \\
\hline Corrected SDRR (ms) & $27.6(17.5)$ & $22.6(8.53)$ & $17.7(9.78)$ & 0.127 \\
\hline RMSSD (ms) & $90.7(58.4)$ & $76.5(39.3)$ & $57.6(35.1)$ & 0.482 \\
\hline NN50 & $138(67.0)$ & $144(77.1)$ & $105(65.5)$ & 0.820 \\
\hline pNN50 & $43.2(23.1)$ & $41.7(24.1)$ & $28.2(18.9)$ & 0.712 \\
\hline LF normalized & $41.4(17.7)$ & $43.2(18.2)$ & 48.5 (18.5) & 0.839 \\
\hline HF normalized & $58.6(17.7)$ & $56.8(18.2)$ & $51.5(18.5)$ & 0.839 \\
\hline LF:HF ratio & $0.93(0.85)$ & $1.06(1.1)$ & $1.3(1.11)$ & 0.820 \\
\hline
\end{tabular}

$P$ values were determined using one-way ANOVA and chi-squared. Prehypertension defined as SBP percentile $>90$ th and $<95$ th. Hypertension defined as SBP percentile $\geq 95^{\text {th }}$. Values presented are mean (SD) or $N(\%)$ where indicated.

DBP, diastolic blood pressure; SBP, systolic blood pressure; SDRR, SD between R-R intervals; RMSSD, root mean square of the square difference between adjacent normal R-R intervals.

(5). Despite this observation, data from cross sectional studies have shown associations of BMI (27), waist circumference (28), skin-fold measured body fat (29), and intra-abdominal fat with higher SBP (30). Data from the current study suggest that, while adiposity may play a role in blood pressure regulation, other physiological factors, such as cANS function, may have a more prominent influence.
At this time it is unclear which physiological mechanisms are responsible for the differential relationships between timedomain and frequency-domain HRV measures and higher SBP in youth. It is possible the difference could be explained by body position, as our measurement under were taken under supine conditions, as frequency-domain perturbations are often elicited under conditions which modulate baroreflexes 
Table 2. Odds ratios for prehypertensive/hypertensive vs. normotensive per unit difference in each HRV measure adjusted for Tanner stage, race, and total body fat

\begin{tabular}{lccc}
\hline & Odds ratio $(95 \% \mathrm{Cl})$ & Standardized coefficient (log odds scale) & $P$ value \\
\hline Lower mean R-R (per 50 ms) & $1.33(1.13,1.57)$ & 3.43 & $<.001$ \\
Lower SDRR (per 10 ms) & $1.11(1.00,1.22)$ & 2.00 & 0.045 \\
Lower corrected SDRR (per 10 ms) & $1.46(1.11,1.92)$ & 2.68 & 0.007 \\
Lower RMSSD (per 10 ms) & $1.11(1.02,1.20)$ & 1.39 & 0.011 \\
Lower NN50 (per 10 units) & $1.04(0.98,1.10)$ & 2.23 & 0.164 \\
Lower pNN50 (per 10 units) & $1.21(1.02,1.44)$ & 1.82 & 0.026 \\
Higher LF normalized (per 10 units) & $1.20(0.99,1.47)$ & -1.82 & 0.069 \\
Higher HF normalized (per 10 units) & $0.83(0.68,1.01)$ & 2.53 & 0.069 \\
Higher LF:HF ratio & $1.47(1.09,1.98)$ & 0.011 \\
\hline
\end{tabular}

Data were analyzed using logistic regression models with prehypertension/hypertension as the outcome, with adjustment made for Tanner stage, race, and total body fat. These models were not adjusted for age, sex, or height since systolic blood pressure percentiles are already adjusted for these variables.

HF, high frequency; HRV, heart rate variability; LF, low frequency; SDRR, SD between R-R intervals; RMSSD, root mean square of the square difference between adjacent normal R-R intervals.

Table 3. Mean differences in systolic blood pressure per unit difference in HRV measures adjusted for Tanner stage, race age, sex, height, and total body fat

\begin{tabular}{|c|c|c|c|}
\hline & $\begin{array}{l}\text { Mean difference } \\
\quad(95 \% \mathrm{Cl})\end{array}$ & $\begin{array}{l}\text { Standardized } \\
\text { coefficient }\end{array}$ & $P$ value \\
\hline $\begin{array}{l}\text { Lower mean R-R } \\
\text { (per } 50 \mathrm{~ms} \text { ) }\end{array}$ & $0.86(0.25,1.48)$ & 2.76 & 0.006 \\
\hline Lower SDRR (per $10 \mathrm{~ms}$ ) & $0.50(0.14,0.87)$ & 2.72 & 0.007 \\
\hline $\begin{array}{l}\text { Lower corrected SDRR } \\
\text { (per } 10 \mathrm{~ms} \text { ) }\end{array}$ & $1.25(0.35,2.15)$ & 2.72 & 0.007 \\
\hline $\begin{array}{l}\text { Lower RMSSD } \\
\text { (per } 10 \mathrm{~ms} \text { ) }\end{array}$ & $0.40(0.14,0.65)$ & 3.07 & 0.002 \\
\hline $\begin{array}{l}\text { Lower NN50 } \\
\text { (per } 10 \text { units) }\end{array}$ & $0.27(0.05,0.49)$ & 2.39 & 0.017 \\
\hline $\begin{array}{l}\text { Lower pNN50 } \\
\text { (per } 10 \text { units) }\end{array}$ & $0.96(0.28,1.64)$ & 2.78 & 0.005 \\
\hline $\begin{array}{l}\text { Higher LF normalized } \\
\text { (per } 10 \text { units) }\end{array}$ & $0.48(-0.44,1.40)$ & 1.02 & 0.309 \\
\hline $\begin{array}{l}\text { Higher HF normalized } \\
\text { (per } 10 \text { units) }\end{array}$ & $-0.48(-1.40,0.44)$ & -1.02 & 0.309 \\
\hline Higher LF:HF ratio & $0.80(-1.48,3.09)$ & 0.69 & 0.491 \\
\hline \multicolumn{4}{|c|}{$\begin{array}{l}\text { Data were analyzed using linear regression with adjustments made for Tanner stage, } \\
\text { race, age, sex, height, and total body fat. } \\
\text { HF, high frequency; HRV, heart rate variability; LF, low frequency; SDRR, SD between R-R } \\
\text { intervals; RMSSD, root mean square of the square difference between adjacent normal } \\
\text { R-R intervals. }\end{array}$} \\
\hline
\end{tabular}

(i.e., standing, head-up or head-down tilt) (31-33). Another potential explanation is that our analysis utilized both continuous and dichotomous classifications of blood pressure, and while some dichotomous associations (LF:HF) were found between frequency-domain HRV measures and classification of hypertension phenotypes, these associations were not robust in continuous models. Perhaps the relatively low sensitivity often observed within some HRV measures (34) might be attributed to the lack of association between higher SBP with frequency-domain HRV measures.

Our study has many strengths including a cohort with a wide range in age, adiposity, pubertal status, and prehypertension/hypertension status (25\% meeting this threshold).
However, it should be noted that our study was cross-sectional in nature, which precludes us from addressing causality. Also, the corrected SDRR used in some of our analyses, has yet to be formally evaluated in pediatrics for validity. Furthermore, blood pressure was measured at a single timepoint (24-h ambulatory blood pressure monitoring was not performed), we were unable to account for the effect of physical activity or fitness, and measures of history of abuse or adverse childhood events were not taken in the present study.

\section{Conclusion}

In conclusion, we have shown for the first time that cANS function (using multiple measures of HRV), independent of adiposity, is significantly associated with prehypertension/ hypertension, and higher levels of SBP among youth. These findings are consistent with the hypothesis that increased sympathetic tone or decreased parasympathetic activity at rest creates a deleterious scenario leading to hypertension in youth which is not necessarily mediated by adiposity. Whether interventions or treatments leading to improvements in sympathetic nervous system activation reduce blood pressure in obese youth independent of weight loss requires further investigation.

\section{METHODS}

\section{Study Design and Participants}

Children and adolescents $(n=188)$, aged $6-18$ y old ( 103 females/85 males), were included in this study. These children and adolescents were participants in a cross-sectional study examining cardiovascular risk factors in youth ranging from normal-weight to severe obesity. Youth with severe obesity were recruited from the University of Minnesota Masonic Children's Hospital Pediatric Weight Management Clinic and other participants were recruited from the community. Participants were excluded if they were taking medications known to influence cardiovascular function or had known/ diagnosed CVD. The study protocol was approved by the University of Minnesota Institutional Review Board, and consent/ assent was obtained from parents/participants.

\section{Anthropometrics, Body Composition Assessment, and Pubertal} Maturation

All testing was performed in the morning after the participants had been fasting (including no caffeine consumption) for a minimum of 
$12 \mathrm{~h}$. Height and weight were determined using a wall-mounted stadiometer and an electronic scale, respectively. BMI was calculated as the body weight in kilograms divided by the height in meters squared. BMI percentiles were determined using age- and gender-based definitions from the Centers for Disease Control and Prevention. Normalweight was defined as $\geq 5$ th to $<85$ th percentile, overweight/obesity was defined as $\geq 85$ th to $<120 \%$ of the 95 th percentile, and severe obesity was defined as $\geq 120 \%$ of the 95 th percentile or an absolute BMI $\geq 35 \mathrm{~kg} / \mathrm{m}^{2}$ (35). Total and regional body composition was measured using DXA (Lunar iDXA, GE Healthcare, Madison, WI) and analyzed using enCore software (platform version 13.6, GE Healthcare). Participants were scanned using standard imaging and positioning protocols while in the fasted state. Tanner stage was determined by a trained pediatrician or nurse $(36,37)$.

\section{Blood Pressure}

Seated BP and HR were measured after the participant had been resting quietly without legs crossed for $10 \mathrm{~min}$. BP and HR were measured three consecutive times with an automated BP cuff at $\sim 3$-min intervals. The average of the three respective BP and HR measurements was used. SBP percentile was determined from age, sex, and height derived from the Fourth Report on the Diagnosis, Evaluation, and Treatment of High Blood Pressure in Children and Adolescents (38). Prehypertension was defined as SBP percentile $\geq 90$ th and $<95$ th and hypertension was defined as SBP percentile $\geq 95$ th.

\section{HRV}

HRV was measured as previously described $(15,39)$, using the SphygmoCor MM3 system (AtCor Medical, Sydney, Australia) after participants had been at rest in a supine position for approximately $15 \mathrm{~min}$. The electrocardiogram signal was then continuously recorded for $5 \mathrm{~min}$; the segment was then reviewed for ectopic heart beats or arrhythmias with any portions of the 5-min segment with abnormal electrocardiogram signals being excluded from analysis.

Automated algorithms were used to calculate time-domains of mean R-R interval length (mean R-R), the SDRR, RMSSD, the number of adjacent N-N intervals over $50 \mathrm{~ms}$ (NN50), and the percentage of adjacent $\mathrm{N}-\mathrm{N}$ intervals over $50 \mathrm{~ms}$ (pNN50). SDRR was also corrected for resting HR due to its confounding influence on SDRR, using an equation developed by Monfredit et al. (40), where corrected $\mathrm{SDRR}=\mathrm{SDRR} / \mathrm{e}^{\mathrm{HR} / 58.8}$. Spectral analysis was used to calculate frequency-domains of LF, HF, the LF to HF (LF:HF) ratio, and total power. LF was defined as frequencies between $0.04-0.15 \mathrm{~Hz}$ and $\mathrm{HF}$ was defined as frequencies between $0.15-0.40 \mathrm{~Hz}$. LF was normalized using the following equation: $\mathrm{LF} /$ (total power-very low frequency) $\times 100$. HF was normalized using the following equation: $\mathrm{HF} /$ (total power-very low frequency) $\times 100$.

\section{Statistical Analysis}

Descriptive statistics were calculated by hypertension group and included means with standard deviations for continuous variables and frequencies with percentages for categorical variables. $P$ values included in Table 1 were based on ANOVA or chi-squared tests for continuous and categorical variables, respectively. All of the regression models used generalized estimating equations with an exchangeable working correlation structure to account for potential correlation between siblings within a family ( 28 families with 2 siblings, 6 with 3 siblings, and 1 with 4 siblings). Robust variance estimation was used for all confidence intervals and $P$ values. For categorical analysis, prehypertension and hypertension were defined as SBP at or above the age, gender, and height specific 90th and 95th percentiles, respectively (38). For regression models with prehypertension/hypertension as the outcome, logistic regression was used and adjusted for Tanner stage, race, and total body fat. These models were not adjusted for age, sex, or height since SBP percentiles are already adjusted for these variables. For continuous analysis, using SBP as the outcome, linear regression was used and adjusted for Tanner stage, race, age, sex, height, and total body fat. All analyses were conducted using R v3.1.1 and the "gee" library v4.13-18.

\section{ACKNOWLEDGMENTS}

The authors thank all of the children and adolescents who participated in this study. The authors also thank Annie Sheldon for her excellent coordination of this study, Cameron Naughton for program management, and Nicholas Evanoff for his technical expertise in measuring heart rate variability. No payment was received to produce this manuscript. All authors have seen, approved, and take full responsibility for the manuscript. J.R.R., D.R.D., K.D.R., and A.S.K. developed the aims and hypothesis. A.S.K. provided the funding. K.D.R. and M.O. performed the statistical analysis. J.R.R. wrote the first draft. M.O., T.A.B., L.C., K.D.R., D.R.D., C.K.F., J.S., and A.S.K. provided subject matter expertise and a critical review of the manuscript.

\section{STATEMENT OF FINANCIAL SUPPORT}

Funding for this project was provided by funding from the National Institutes of Health ( $\mathrm{NIH}$; Bethesda, MD) the National Heart, Lung, and Blood Institute/NIH (R01HL110957, awarded to A.S.K.), the National Center for Advancing Translational Sciences/NIH (UL1TR000114), National Institute of Diabetes and Digestive and Kidney Diseases (NIDDK)/NIH NORC Grant Number P30 DK050456, and a training grant from the NIDDK/NIH (T32-DK083250 to J.R.R).

Disclosure: A.S.K. serves on pediatric obesity advisory boards (Novo Nordisk; Takeda) but does not accept personal or professional income for his services.

\section{REFERENCES}

1. Gu Q, Burt VL, Paulose-Ram R, Yoon S, Gillum RF. High blood pressure and cardiovascular disease mortality risk among U.S. adults: the third National Health and Nutrition Examination Survey mortality follow-up study. Ann Epidemiol 2008;18:302-9.

2. Juhola J, Magnussen CG, Viikari JS, et al. Tracking of serum lipid levels, blood pressure, and body mass index from childhood to adulthood: the Cardiovascular Risk in Young Finns Study. J Pediatr 2011;159:584-90.

3. Rapsomaniki E, Timmis A, George J, et al. Blood pressure and incidence of twelve cardiovascular diseases: lifetime risks, healthy life-years lost, and age-specific associations in 1.25 million people. Lancet 2014;383: 1899-911.

4. Singh M, Mensah GA, Bakris G. Pathogenesis and clinical physiology of hypertension. Cardiol Clin 2010;28:545-59.

5. Freedman DS, Goodman A, Contreras OA, DasMahapatra P, Srinivasan SR, Berenson GS. Secular trends in BMI and blood pressure among children and adolescents: the Bogalusa Heart Study. Pediatrics 2012;130:e159-66.

6. Joyner MJ, Charkoudian N, Wallin BG. Sympathetic nervous system and blood pressure in humans: individualized patterns of regulation and their implications. Hypertension 2010;56:10-6.

7. Electrophysiology of heart rate variability: standards of measurement, physiological interpretation, and clinical use. Circulation 1996; 93: 1043-1065.

8. Heart rate variability: standards of measurement, physiological interpretation and clinical use. Task Force of the European Society of Cardiology and the North American Society of Pacing and Electrophysiology. Circulation 1996; 93:1043-1065.

9. Saul JP, Rea RF, Eckberg DL, Berger RD, Cohen RJ. Heart rate and muscle sympathetic nerve variability during reflex changes of autonomic activity. Am J Physiol 1990;258(3 Pt 2):H713-21.

10. Malliani A, Pagani M, Lombardi F, Cerutti S. Cardiovascular neural regulation explored in the frequency domain. Circulation 1991;84:482-92.

11. Tsuji H, Larson MG, Venditti FJ Jr, et al. Impact of reduced heart rate variability on risk for cardiac events. The Framingham Heart Study. Circulation 1996;94:2850-5.

12. Dekker JM, Crow RS, Folsom AR, et al. Low heart rate variability in a 2-minute rhythm strip predicts risk of coronary heart disease and mortality from several causes: the ARIC Study. Atherosclerosis Risk In Communities. Circulation 2000;102:1239-44.

13. Henje Blom E, Olsson EM, Serlachius E, Ericson M, Ingvar M. Heart rate variability is related to self-reported physical activity in a healthy adolescent population. Eur J Appl Physiol 2009;106:877-83. 


\section{Articles Ryderetal.}

14. Gutin B, Howe C, Johnson MH, Humphries MC, Snieder H, Barbeau P. Heart rate variability in adolescents: relations to physical activity, fitness, and adiposity. Med Sci Sports Exerc 2005;37:1856-63.

15. Kaufman CL, Kaiser DR, Steinberger J, Dengel DR. Relationships between heart rate variability, vascular function, and adiposity in children. Clin Auton Res 2007;17:165-71.

16. Farah BQ, Barros MV, Balagopal B, Ritti-Dias RM. Heart rate variability and cardiovascular risk factors in adolescent boys. J Pediatr 2014;165:945-50.

17. Norris AL, Steinberger J, Steffen LM, Metzig AM, Schwarzenberg SJ, Kelly AS. Circulating oxidized LDL and inflammation in extreme pediatric obesity. Obesity (Silver Spring) 2011;19:1415-9.

18. Gidding SS, Nehgme R, Heise C, Muscar C, Linton A, Hassink S. Severe obesity associated with cardiovascular deconditioning, high prevalence of cardiovascular risk factors, diabetes mellitus/hyperinsulinemia, and respiratory compromise. J Pediatr 2004;144:766-9.

19. Tryggestad JB, Thompson DM, Copeland KC, Short KR. Obese children have higher arterial elasticity without a difference in endothelial function: the role of body composition. Obesity (Silver Spring) 2012;20:165-71.

20. Martini G, Riva P, Rabbia F, et al. Heart rate variability in childhood obesity. Clin Auton Res 2001;11:87-91.

21. Rabbia F, Silke B, Conterno A, et al. Assessment of cardiac autonomic modulation during adolescent obesity. Obes Res 2003;11:541-8.

22. Riva P, Martini G, Rabbia F, et al. Obesity and autonomic function in adolescence. Clin Exp Hypertens 2001;23:57-67.

23. Hillebrand S, de Mutsert R, Christen T, et al.; NEO Study Group. Body fat, especially visceral fat, is associated with electrocardiographic measures of sympathetic activation. Obesity (Silver Spring) 2014;22:1553-9.

24. Chen SR, Chiu HW, Lee YJ, Sheen TC, Jeng C. Impact of pubertal development and physical activity on heart rate variability in overweight and obese children in Taiwan. J Sch Nurs 2012;28:284-90.

25. Fox CS, Massaro JM, Hoffmann U, et al. Abdominal visceral and subcutaneous adipose tissue compartments: association with metabolic risk factors in the Framingham Heart Study. Circulation 2007;116:39-48.

26. Garrison RJ, Kannel WB, Stokes J 3rd, Castelli WP. Incidence and precursors of hypertension in young adults: the Framingham Offspring Study. Prev Med 1987;16:235-51.

27. Paradis G, Lambert M, O'Loughlin J, et al. Blood pressure and adiposity in children and adolescents. Circulation 2004;110:1832-8.

28. Bekkers MB, Brunekreef B, Koppelman GH, et al. BMI and waist circumference; cross-sectional and prospective associations with blood pressure and cholesterol in 12-year-olds. PLoS One 2012;7:e51801.
29. Shear CL, Freedman DS, Burke GL, Harsha DW, Berenson GS. Body fat patterning and blood pressure in children and young adults. The Bogalusa Heart Study. Hypertension 1987;9:236-44.

30. Syme C, Abrahamowicz M, Leonard GT, et al. Intra-abdominal adiposity and individual components of the metabolic syndrome in adolescence: sex differences and underlying mechanisms. Arch Pediatr Adolesc Med 2008;162:453-61.

31. Bloomfield DM, Kaufman ES, Bigger JT Jr, Fleiss J, Rolnitzky L, Steinman R. Passive head-up tilt and actively standing up produce similar overall changes in autonomic balance. Am Heart J 1997;134(2 Pt 1): 316-20.

32. Taylor JA, Carr DL, Myers CW, Eckberg DL. Mechanisms underlying very-low-frequency RR-interval oscillations in humans. Circulation 1998;98:547-55.

33. Bosch TA, Kaufman CL, Williamson EB, Duprez DA, Dengel DR. Comparison of changes in heart rate variability and blood pressure during nitroglycerin administration and head-up tilt testing. Clin Auton Res 2009;19:46-50.

34. Kleiger RE, Stein PK, Bigger JT Jr. Heart rate variability: measurement and clinical utility. Ann Noninvasive Electrocardiol 2005;10:88-101.

35. Kelly AS, Barlow SE, Rao G, et al.; American Heart Association Atherosclerosis, Hypertension, and Obesity in the Young Committee of the Council on Cardiovascular Disease in the Young, Council on Nutrition, Physical Activity and Metabolism, and Council on Clinical Cardiology. Severe obesity in children and adolescents: identification, associated health risks, and treatment approaches: a scientific statement from the American Heart Association. Circulation 2013;128:1689-712.

36. Marshall WA, Tanner JM. Variations in the pattern of pubertal changes in boys. Arch Dis Child 1970;45:13-23.

37. Marshall WA, Tanner JM. Variations in pattern of pubertal changes in girls. Arch Dis Child 1969;44:291-303.

38. The fourth report on the diagnosis, evaluation, and treatment of high blood pressure in children and adolescents. Pediatrics 2004; 114:555576.

39. Kelly AS, Rudser KD, Dengel DR, et al. Cardiac autonomic dysfunction and arterial stiffness among children and adolescents with attention deficit hyperactivity disorder treated with stimulants. J Pediatr 2014;165: 755-9.

40. Monfredi O, Lyashkov AE, Johnsen AB, et al. Biophysical characterization of the underappreciated and important relationship between heart rate variability and heart rate. Hypertension 2014;64:1334-43. 